\title{
A rare case of Addison's disease, hepatitis, thyreoiditis, positive IgG anti-tissue transglutaminase antibodies and partial IgA deficiency
}

\author{
MARTA P. BALEVA ${ }^{l}$, SNEJINA MIHAYLOVA ${ }^{l}$, PETJA YANKOVA ${ }^{l}$, ILIANA ATANASOVA ${ }^{2}$, \\ MILENA NIKOLOVA-VLAHOVA ${ }^{3}$, ELISSAVETA NAUMOVA ${ }^{l}$ \\ ${ }^{1}$ Department of Clinical Immunology, University Hospital Alexandrovska, Medical University-Sofia, Sofia, Bulgaria \\ ${ }^{2}$ University Hospital for Endocrinology, Medical University-Sofia, Sofia, Bulgaria \\ ${ }^{3}$ Department of Nephrology, University Hospital Alexandrovska, Medical University-Sofia, Sofia, Bulgaria
}

\begin{abstract}
Introduction: Selective IgA deficiency (IgAD) is the most prevalent type of primary immune deficiencies, but partial IgA deficiency is even more common. Addison's disease is a rare condition associated with primary adrenal insufficiency due to infection or autoimmune destruction of the adrenals. The association between IgA deficiency and Addison's disease is very rare.

Case and laboratory data: We observed a 22-year-old male patient with marked darkening of the skin, especially on the palms and areolae, jaundice on the skin and sclera, astheno-adynamia, hypotension $(80 / 50 \mathrm{~mm} \mathrm{Hg})$, and pain in the right hypochondrium. The laboratory investigations revealed increased serum levels of total and indirect bilirubin, AST, ALT, GGT and LDH, negative HBsAg, anti-HBc IgM, anti-HCV and anti-HAV IgM, very low serum IgA levels $(0.16 \mathrm{~g} / \mathrm{l})$ with normal $\mathrm{Ig} G$ and IgM, negative ANA, ANCA, AMA, LKM-1, anti-GAD-60, anti-IA-2, anti-thyroglobulin antibodies, a mild increase in anti-TPO antibodies titer, a marked increase in IgG anti-tissue transglutaminase antibodies, with no typical changes in cellular immunity, negative T-SPOT-TB test, $H L A-A * 01 ; B * 08 ; D R B 1 * 03$; $D Q B 1 * 02$, karyotype $-46, X Y$.

Conclusions: We present a rare case of partial IgA deficiency with Addison's disease, hepatitis, thyroiditis and positive anti-tissue transglutaminase antibodies. IgAD and some autoimmune disorders share several predisposing HLA genes, thus explaining the increased prevalence of IgAD in certain patient groups.
\end{abstract}

Key words: Addison's disease, partial IgA deficiency, thyreoiditis, tissue transglutaminase antibodie.

(Cent Eur J Immunol 2016; 41 (2): 217-220)

\section{Introduction}

Selective $\operatorname{Ig}$ A deficiency (IgAD) is the most prevalent type of primary immune deficiencies. Serum $\operatorname{IgA}$ level of less than $0.07 \mathrm{~g} / \mathrm{l}$ with normal values of $\operatorname{IgG}$ and $\operatorname{IgM}$ is considered as selective IgA deficiency in patients older than 4 years. If the serum IgA level is higher than this value but two standard deviations below the normal for age, the condition is described as partial IgA deficiency [1]. Selective IgA deficiency has variable prevalence in different ethnic groups. The lowest prevalence is reported among Japanese blood donors - from $1: 14840$ to $1: 18500$ [2]. In Caucasian blood donors it varies from $1: 300$ to $1: 1200$ [3]. Partial IgA deficiency is even more common [1].
Most persons with IgAD are asymptomatic [1]. Serum $\mathrm{IgAD}$ is usually associated with recurrent respiratory tract infections (in approximately half of the affected), autoimmune diseases (in 28\%), asthma and allergic diseases and conditions (in 13\%) [4]. There are a few publications on the association between serum IgAD and Addison's disease (AD).

\section{Case report}

We observed a 22-year-old male patient with marked darkening of the skin, especially on the palms and areolae, jaundice on the skin and sclera, astheno-adynamia, hypotension $(80 / 50 \mathrm{~mm} \mathrm{Hg})$, pain in the right hypochondrium.

Correspondence: Marta P. Baleva, Department of Clinical Immunology, University Hospital Alexandrovska, Medical University-Sofia, 1. G. Sofiiski str., 1431 Sofia, Bulgaria, e-mail: marta_baleva@yahoo.com Submitted: 14.07.2015, Accepted: 12.10.2015 
The physical examination revealed asthenic constitution, height $180 \mathrm{~cm}$, body weight $55 \mathrm{~kg}$, reduced facial and body hairs, reduced subcutaneous fat tissue, jaundice on the skin and visible linings, heart rate $105 \mathrm{bpm}$, enlarged liver, brownish spots on the gums and on the hands. The patient reported that several years ago he was treated with local antimycotic agents for alopecia areata and subsequently received local antimycotics for perioral rash and oral lesions.

The clinical-laboratory investigations revealed increased serum levels of total and indirect bilirubin, AST, ALT, GGT and LDH, low serum sodium and chlorides, but normal potassium, a mild increase in FT4 with increased TSH, very high morning ACTH levels with serum morning cortisol below the normal ranges, and normal testosterone. Whole blood count, biochemical studies, coagulation, glucose, iron, copper, iron-binding capacity, urine sediment, were normal at baseline and during the follow-up. The patient was HBsAg, anti-HBc IgM, anti-HCV and anti-HAV IgM negative.

The immunological investigations showed very low serum IgA levels $(0.16 \mathrm{~g} / \mathrm{l})$ with normal $\mathrm{IgG}$ and $\mathrm{IgM}$, negative ANA, ANCA, AMA, LKM-1 antibodies, antiGAD-60, anti-IA-2, anti-thyroglobulin antibodies, a mild increase in anti-TPO antibodies titer, a marked increase in IgG anti-tissue transglutaminase antibodies - $200 \mathrm{U} / \mathrm{ml}$

Table 1. Biochemical results and immunological investigations of the patient

\begin{tabular}{lcc}
\hline \multicolumn{1}{c}{ Parameter } & $\begin{array}{c}\text { Patient's } \\
\text { value }\end{array}$ & Normal values \\
\hline Total serum bilirubin & 43 & $3.4-21 \mu \mathrm{mol} / \mathrm{l}$ \\
\hline Direct serum bilirubin & 8.6 & $0.8-8.5 \mu \mathrm{mol} / \mathrm{l}$ \\
\hline Serum AST & 49 & $5-40 \mathrm{U} / \mathrm{l}$ \\
\hline Serum ALT & 152 & $5-40 \mathrm{U} / 1$ \\
\hline Serum GGT & 623 & $<50 \mathrm{U} / 1$ \\
\hline Thyroid stimulating hormone & 8.6 & $0.35-5.5 \mathrm{mIU} / 1$ \\
\hline Serum FT4 & 21.37 & $9-20 \mathrm{pmol} / 1$ \\
\hline Plasma morning ACTH & 1880 & $8-66 \mathrm{pg} / \mathrm{ml}$ \\
\hline Serum morning cortisol & 62.9 & $124-662 \mathrm{nmol} / 1$ \\
\hline Serum sodium & 100 & $136-151 \mathrm{mmol} / 1$ \\
\hline Serum chlorides & 66 & $96-110 \mathrm{mmol} / 1$ \\
\hline Serum potassium & 4.2 & $3.5-5.6 \mathrm{mmol} / 1$ \\
\hline IgG & 13.35 & $5.4-16.1 \mathrm{~g} / 1$ \\
\hline IgM & 0.85 & $0.5-2 \mathrm{~g} / \mathrm{l}$ \\
\hline IgA & 0.16 & $0.8-2.8 \mathrm{~g} / 1$ \\
\hline $\begin{array}{l}\text { Anti-thyroid peroxidase } \\
\text { antibodies }\end{array}$ & 42 & $0-34 \mathrm{mU} / 1$ \\
\hline $\begin{array}{l}\text { IgG anti-tissue } \\
\text { transglutaminase }\end{array}$ & 200 & $<15 \mathrm{U} / \mathrm{ml}$ \\
\hline & & \\
\hline
\end{tabular}

(Table 1), with no changes in cellular immunity (Th, Ts, $B$ and NK cells with normal CD4/CD8 ratio), negative T-SPOT-TB test; HLA type - A*01; B*08; DRB $1 * 03$; DQB1*02; karyotype - 46, XY.

The thyroid ultrasound examination revealed normal homogenic glandular parenchyma, thyroid volume $7.8 \mathrm{ml}$. The abdominal ultrasound failed to visualize the adrenal glands. The liver biopsy showed no inflammation, no fibrosis.

The computed tomography (CT) of the brain revealed normal position and symmetrical narrowing of the ventricular system, narrowing of the subarachnoid spaces, normal hypothalamus and pituitary gland.

The patient was diagnosed with partial serum IgA deficiency together with primary hypocorticism (Addison's disease) based on typical skin darkening, astheno-adynamia, hypotension, very high ACTH levels with low morning serum cortisol, no CT changes in the hypothalamus and pituitary gland. He was treated with saline and glucose infusions, Mannitol, Famotidine, intravenous glucocorticosteroids (methylprednisolone 60-80 mg per day), Fludrocortisone $0.1 \mathrm{mg}$. The patient was discharged with improvement and remained on the following treatment: Dehydrocortisone $5 \mathrm{mg} 1+1 / 4$ tablet, Fludrocortisone 0.1 mg $1 / 2$ tablet every morning (1/4 tablet in winter months).

\section{Discussion}

In 1855, Thomas Addison described an unknown condition with "anemia, general languor and debility, remarkable feebleness of the heart's action, irritability of the stomach and peculiar change of color of the skin, occurring in connection with a diseased condition of the suprarenal capsules" [5]. In 1856, A. Trousseau called the adrenocortical insufficiency "Addison's disease" [6]. The symptom complex described by T. Addison is thought to be the first case of autoimmune adrenalitis as a separate disease entity. Adrenocortical insufficiency could be primary (due to destruction of the adrenal glands) or secondary (due to pituitary and/or hypothalamic disease). In the past, the major cause of the disease was tuberculosis, and in the past 4050 years the main pathogenetic mechanism is autoimmune adrenalitis. Addison's disease could be a separate disease entity or a part of autoimmune polyglandular syndromes (APS) type I, II or IV: type I (AD, chronic candidiasis, chronic hypoparathyroidism - at least 2/3); type II (AD, autoimmune thyroid diseases, type I diabetes mellitus); type IV (two or more organ specific autoimmune diseases, which do not fall into previous types) [7, 8]. Type III APS does not include AD [7].

Betterle et al. [5] described the most frequent organ-specific autoimmune diseases and conditions that accompany AD in 1240 European patients. According to their data, chronic hepatitis is found in $1.6-3 \%$ of $\mathrm{AD}$ patients. In APS I the prevalence of chronic active hepati- 
tis is $5-31 \%$ [6]. In these patients, a transitory increase in serum aminotransferase levels is found and some of the affected have positive LKM-1 antibodies [7]. In APS type II chronic hepatitis is found in a lower percentage of the patients $-4 \%$, there are no data concerning the prevalence of chronic hepatitis in APS type IV [6]. In our patient, the disease onset was 1-2 years before the appearance of the classical AD symptoms, with alopecia areata, perioral rash and changes in oral lining treated successfully with antimycotics. Later the patient was admitted for Addison crisis. He had increased aminotransferase total and indirect bilirubin levels but ANA, antimitochondrial, LKM-1 antibodies were negative, as were the serological markers for hepatitis A, B and C. Abdominal ultrasound and liver biopsy showed no pathological findings.

Addison's disease is frequently associated with autoimmune thyroid diseases - Hashimoto's thyroiditis (3.7$32 \%$ ) and Graves' disease (2-22.7\%), and $1.2-8 \%$ of patients have celiac disease [6]. Our patient had laboratory data for autoimmune thyroid disease with mild changes in FT4, TSH and anti-TPO antibodies, but without ultrasound changes in the thyroid gland. The patient reported no gastro-intestinal symptoms but IgG anti-tissue transglutaminase antibodies were positive (Table 1). In the available medical literature we found two reports of the association of AD with celiac disease and IgAD. In 1997, Heneghan et al. [9] found 2 cases of AD and selective IgA deficiency among 700 patients with biopsy-proven celiac disease. In 2006, Betterle et al. [10] found clinical, silent or latent forms of celiac disease in 6/109 (5.4\%) of the investigated AD patients ( 1 - with APS type I, 4 - with APS type II, 1 - with AD). Two of these patients had serum IgAD and all had normal anti-gliadin antibody levels. In patients with negative $\operatorname{IgA}$ anti-tissue transglutaminase antibody levels and suspected celiac disease serum IgA levels should be investigated and if the total $\operatorname{IgA}$ is low, IgG anti-tissue transglutaminase should be evaluated [11]. Our patient was recommended dietary changes with exclusion of gluten because of the risk of future development of malabsorption associated with $\mathrm{AD}$ and celiac disease. Like O'Leary et al. [12], we share the opinion that the detrimental effect of substation treatment for AD on the patient's gastro-intestinal tract should not be underestimated because this could lead to further worsening of the patient's condition.

No changes in immunophenotyping of blood lymphocytes were found. T-SPOT-TB was negative and this practically excludes the tuberculosis as a cause of AD.

The low IgA serum levels $(0.16 \mathrm{~g} / \mathrm{l}$, normal range 0.8 $2.8 \mathrm{~g} / 1)$ classify our patient as having partial IgAD [1]. The selective IgAD is underestimated due to the cut-off value of $0.07 \mathrm{~g} / 1$ that distinguishes partial from selective $\mathrm{IgAD}$. It remains disputable whether the patients with partial IgAD are more prone to infections and autoimmune diseases. Shakkottai et al. [13] describe spondyloarthropathy, Sjögren's syndrome, Raynaud's syndrome, joint pain and edema, morning stiffness, frequent infections, allergies and eczema in children with partial $\operatorname{IgAD}$, and in some of them - positive ANA ( $1: 80$ to $1: 320)$ can be detected.

IgA deficiency is characterized by a defect of terminal lymphocyte differentiation, leading to lack of IgA in serum and mucosal secretions. A strong association between $\mathrm{Ig} \mathrm{AD}$ and autoimmune diseases has been widely described. Both major histocompatibility complex (MHC) and nonMHC genes contribute to susceptibility to the disease. Interestingly, the ancestral 8.1. haplotype is also reported to be associated with Grave's disease, systemic lupus erythematosus, type I diabetes and celiac disease $[14,15]$. It is therefore possible that IgAD and some autoimmune disorders share some of the predisposing genes, thus explaining the increased prevalence of IgAD in certain patient groups. On the other hand, the common HLA genotype in autoimmune conditions and in IgAD poses a question whether IgAD itself is an autoimmune disease. The HLA type of our patient was: A*01; B*08; DRB1*03; DQB1*02. The most frequent haplotype in patients with $\operatorname{IgAD}$ is haplotype 8.1. (HLA - A1; B8; DR3; DQ2) that is detected in $45 \%$ of the cases compared with $16 \%$ of the general population [3]. A high prevalence of HLA-DR3 is found in isolated AD as well [5]. HLA-DRB1*0301, DQA1*0501 DQB $1 * 0201$ have been described in APS II [5].

On the other hand, the possibility of coexistence of IgAD and autoimmune polyglandular syndrome (APS) type II should not be excluded in this patient. APS type II is a relatively common autoimmune disease, affecting approximately $1-2$ patients $/ 10000$ a year with a female : male ratio of $3: 1[5,8,10,16]$. APS type II manifests from childhood to early adulthood with autoimmune thyroid disease, type I diabetes, Addison's disease, hypoparathyroidism, hypopituitarism, typically without chronic candida infections [16]. Cases of autoimmune endocrinopathies and selective IgAD have been described [10, 17]. Of particular importance in our patient is the possibility for development of autoimmune diabetes (type 1 diabetes mellitus) and, therefore, dynamic follow-up of glucose metabolism is needed.

The association of IgAD and Addison's disease is rare. In the described patient these two conditions are associated with several autoimmune diseases - hepatitis, thyroiditis and celiac disease. The hepatitis was presumed autoimmune as no causative viral, metabolic or toxic agent was found, despite the negative anti-liver antibodies. The thyroiditis was considered of anti-TPO type (Hashimoto thyroiditis). Moreover, the patient had serological data for celiac disease - positive IgG anti-tissue transglutaminase levels. We support the thesis of A. Shakkottai et al. [13] that in patients with celiac disease with normal $\operatorname{IgA}$ antitissue transglutaminase levels, the IgG subtype of these autoantibodies should be investigated and selective or partial IgAD should be sought. 
The authors declare no conflict of interest.

\section{References}

1. Yel L (2010): Selective IgA deficiency. J Clin Immunol 30: 10-16.

2. Kanoh T, Mizumoto T, Yasuda N, et al. (1986): Selective IgA deficiency in Japanese blood donors. Frequency and statistical analysis. Vox Sang 50: 81-86.

3. Singh K, Chang C, Gershwin ME (2014): IgA deficiency and autoimmunity. Autoimmunity Rev 13: 163-177.

4. Edwards E, Razvi S, Cunnigham-Rundles C (2004): IgA deficiency: clinical correlates and responses to pneumococcal vaccine. Clin Immunol 111: 93-97.

5. Betterle C, Dal Pra C, Mantero F, Zanchetta R (2002): Autoimmune adrenal insufficiency and autoimmune polyendocrine syndromes: autoantibodies, autoantigens, and their applicability in diagnosis and disease prediction. Endocrinol Rev 23: 327-364.

6. Trousseau A (1896): Bronze Addison's disease. Arch Gen Med 8: 478-485.

7. Neufeld M, Blizzard RM. Polyglandular autoimmune diseases. In: Symposium on autoimmune aspects of endocrine disorders. Pinchera A, Doniach D, Fenzi GF, Baschieri L (eds.). New York Academic Press, New York 1980; 357-365.

8. Betterle C, Greggio NA, Volpato M (1998): Autoimmune polyglandular syndrome type I. J Clin Endocrinol Metabolism 83: 1049-1055.

9. Heneghan MA, McHugh P, Stevens FM, McCarthy CE (1997): Addison's disease and selective IgA deficiency in two coeliac patients. Scand J Gastroenterol 32: 509-511.

10. Betterle C, Lazzarotto F, Spadaccino AC, et al. (2006): Celiac disease in North Italian patients with autoimmune Addison's disease. Eur J Endocrinol 154: 275-279.

11. McGowan KE, Lyon ME, Butzner JD (2008): Celiac disease and IgA deficiency: complications of serological testing approaches encounterd in the clinic. Clin Chemistry 54: 12031209.

12. O'Leary C, Walsh CH, Wieneke P, et al. (2002): Coeliac disease and autoimmune Addison's disease: a clinical pitfall. Q J Med 95: 79-82.

13. Shakkottai A, Bupathi K, Patel AP, et al. (2012): Children with partial IgA deficiency: clinical characteristics observed in the pediatric rheumatology clinic. Clin Pediatrics 51: 4650 .

14. Jorgensen GH, Ornolfsson AE, Johannesson A, et al. (2011): Association of immunoglobulin A deficiency and elevated thyrotropin-receptor autoantibodies in two Nordic countries. Hum Immunol 72: 166-172.

15. Price P, Witt C, Allcock R, et al. (1999): The genetic basis for the association of the 8.1 ancestral haplotype (A1, B8, DR3) with multiple immunopathological diseases. Immunol Rev 167: 257-274.

16. Kahaly GJ (2009): Polyglandular autoimmune syndromes. Eur J Endocrinol 161: 11-20.

17. Torrelo A, Espańa A, Balsa J, Ledo A (1992): Vitiligo and polyglandular autoimmune syndrome with selective IgA deficiency. Int J Dermatol 31: 343-344. 